Bull. Chem. Soc. Ethiop. 2017, 31(1), 39-49.

ISSN 1011-3924

(C) 2017 Chemical Society of Ethiopia and The Authors

Printed in Ethiopia

DOI: http://dx.doi.org/10.4314/bcse.v31i1.4

\title{
DIRECT ACETYLATION OF SUNFLOWER OIL IN THE PRESENCE OF BORON TRIOXIDE CATALYST AND THE ADDUCT USAGE AS THE BASE STOCK AND LUBRICANT ADDITIVE
}

\author{
Javad Ashrafi $^{1}$, Abolfazl Semnani ${ }^{1 *}$, Hamid Shakoori Langeroodi ${ }^{2}$ and Mahboube Shirani ${ }^{3}$ \\ ${ }^{1}$ Department of Chemistry, Faculty of Sciences, University of Shahrekord, Shahrekord, Iran \\ ${ }^{2}$ Petrokimia Sepahan Company, Mobareke hIndustrial City, Isfahan, Iran \\ ${ }^{3}$ Department of Chemistry, Faculty of Science, University of Jiroft, Jiroft, Iran
}

(Received July 19, 2014; revised August 15, 2016)

\begin{abstract}
Lubrication properties of sunflower oil have been modified by epoxidation in the first step and acetylation of the obtained epoxide in the second step. Epoxidation has been followed in dichloromethane solution in the presence of hydrogen peroxide and acetic acid as oxidizing agent and sulfuric acid as catalyst. The reaction has been monitored and optimized by measurement of iodine value, epoxy content, and recording the FTIR spectrum at different experimental conditions. Acetylation has been performed by enduring the optimized conditions of epoxidation reaction, in the presence of boron trioxide as catalyst and at elevated temperature. The recent reaction has been monitored and optimized by measurement of hydroxyl value, epoxy content, and recording the FTIR spectrum at different experimental conditions. The final product of both,epoxidation and acetylation reactions has been confirmed by FTIR and NMR data. Relative to sunflower oil, pour point and oxidation stability of the modified oil has considerably been improved. The modified oil has successfully been employed in the formulation of industrial gear oil. The investigation on the possibility of the additive application of the modified oil, as well as its miscibility with additives was considered.
\end{abstract}

KEY WORDS: Sunflower oil, Lubrication, Epoxidation, Acetylation, Borontrioxide, Gear oil blending, Polyalphaolephin-100, Polyisobutene

\section{INTRODUCTION}

Environmental concerns over the use of petroleum-based products in various industries, such as forestry, farming, mining, boating, and others, has led to increased interest in the use of environmentally friendly fluids. In addition, the petroleum sources are limited and it is necessary to find alternatives for them. The beneficial aspects of vegetable oils as lubricants are mainly their biodegradability and non-toxicity, which are not exhibited by conventional mineral base oils. Their volatility is low, because of the high molecular weight of the triglyceride structure and by increasing the temperature their viscosity changes mildly. The ester linkages deliver inherent lubricity on metallic surfaces because of their adhesive property. Further, vegetable oils have superior solubilizing power for contaminants and additive molecules compared with mineral base fluids [1-5]. Because of existing of bis-allylic protons in the fatty acid chain vegetable oils have poor oxidation stability. Increased acidity, corrosion, viscosity, and volatility are the unwanted results of the recent effect. Deposit forming tendency, low temperature solidification and low hydrolytic stability, are the other disadvantages of vegetable oils. The high pour point is one of the other restrictions of vegetable oils, which limits their use in low temperature conditions [6-9]. Chemical modification, genetic modification [10-14], blending with other base oils and development of special additives [16-23] are the different methods which overcomes the noted problems of vegetable oils. In this paper chemical modification by initial epoxidation of sunflower oil, followed by acetylation of epoxidized intermediate has been followed. This research is in continuing of our research on lubricants [2429]. Economic considerations involve chemical modifications to be directed toward catalyst which could be applied at ambient temperature. This aim will be approached by examination of

*Corresponding author. E-mail: a.semnani1341@gmail.com 
different catalysts. In this paper the effect of boron trioxide catalyst on the acetylation of epoxidized oil has been investigated. Based on the best of our knowledge there is no report on the application of this catalyst. Formulation of gear oil by modified adduct as well as examination of its miscibility are the other novel aspect of this work.

\section{EXPERIMENTAL}

\section{Materials}

All of the materials were analytical grade and they were used without any further purification. They were all purchased from Merck Company. Hydrogen peroxide was used for oxidation of sunflower oil. Crystal violet was applied for determination of iodine number. Liquid bromine, acetic acid (96\%), sulfuric acid (98\%), glacial acetic acid, acetic anhydride, pyridine, potassium hydroxide were all purchased from Merck. Other employed materials, their function are given in Table 1.

\section{Test methods}

The test methods were followed as: ASTM D-5558-95 for saponification value, AOCS Ca 3a 63 for acid value, AOCS Cd 1-25 for iodine value, Penn State Microoxidation Kit [30] for oxidation stability, ASTM D-86 for hydroxyl number, AOCS Cd 9-57 for epoxy content, ASTM D-445 for viscosity @ $40{ }^{\circ} \mathrm{C}$ and $100{ }^{\circ} \mathrm{C}$, ASTM D-2270 for viscosity index, ASTM D92 for flash point, ASTM D-97 for pour point, ASTM D-1298 for specific gravity, ASTM D2622 for sulfur content, ASTM D-1500 for color, ASTM D-664 for total acid number, ASTM D-6304 for water content, ASTM D-2501for viscosity gravity constant (VGC), ASTM 5182 for FZG, and ASTM D-2782 for Timken load.

Table 1. List of the used materials, as well as their function and their manufacturer.

\begin{tabular}{|l|l|l|}
\hline Material & Function & Manufacturer \\
\hline Hydrogen peroxide, 99/99\% & Oxidation of sunflower oil & Merck Company \\
Sulfuric acid & Oxidation of sunflower oil & Merck Company \\
Acetic acid & Catalyst for oxidation of sunflower oil & Merck Company \\
Boron trioxide & Ring opening of epoxide & Merck Company \\
Liquid bromine & Catalyst for ring opening of epoxide & Merck Company \\
Carbontetrachloride & Determination of iodine number & Merck Company \\
$33 \%$ Hydrogen bromide in glacial & Determination of iodine number & Merck Company \\
acetic acid & Determination of epoxide content & Merck Company \\
Crystal violet & Determination of epoxide content & Aldrich Company \\
Acetic anhydride & Determination of hydroxyl value & Merck Company \\
Pyridine & Determination of hydroxyl value & Merck Company \\
Alcoholic potassium hydroxide & Determination of hydroxyl value & Merck Company \\
Gear oil Conoco & Reference oil for equalization & Conoco Phllips \\
HiTEC 343 & Additive package for formulation of gear & Afton Company \\
LZ 6665 & oil & \\
Polyisobutene & Pour point depressant & Lubrizol Company \\
Polyalphaolephin & Examination of miscibility with acetylated & \\
oil & ELA Company \\
EN 150 & Examination of miscibility with acetylated & \\
oil & Exon Mobil Company \\
\hline
\end{tabular}




\section{Instrumentation}

The viscosities, viscosity indices and specific gravities of compounds were determined by viscometer Anton bar model SVM 3000. Flash points were evaluated by flash point tester Herzog model HC 852. Pour points were determined by pour point tester Herzog model HC 852. The colors were determined by Dr. Long instrument. FTIR spectra were recorded on a JASCO 6300 FT-IR system in a scanning range of $400-4000 \mathrm{~cm}^{-1}$ for 32 scans at a spectral resolution of $4 \mathrm{~cm}^{-1}$ with a pair of $\mathrm{KBr}$ crystals in thin film. ${ }^{1} \mathrm{H}$ NMR spectra were recorded using a Bruker Avance III 400 NMR, using a $5 \mathrm{~mm}$ broad band inverse Z-gradient probe in $\mathrm{CDCl}_{3}$, and Bruker Icon NMR software was used. Peaks were referenced to 3-tetramethylsilane$\mathrm{d}_{4}$ (TMS) at $0.0000 \mathrm{ppm}$. Each spectrum was Fourier transformed, phase corrected, and integrated using ACD spectrum manager.

Epoxidation of sunflower oil

In a dry three neck $500 \mathrm{~mL}$ round-bottom flask fitted with a condenser and equipped with a thermometer, $15 \mathrm{~mL}$ of sunflower oil, $10 \mathrm{~mL}$ of glacial acetic acid and $5 \mathrm{~mL}$ of dichloromethane were mixed. To the recent mixture, $10 \mathrm{~mL}$ of $\mathrm{H}_{2} \mathrm{O}_{2} 30 \%$ was added drop by drop. In the next step, $0.1 \mathrm{~mL}$ of $\mathrm{H}_{2} \mathrm{SO}_{4}$ catalyst was added to the blend. While stirring, the mixture was heated at $60{ }^{\circ} \mathrm{C}$ for two hours, the muddle was cooled to room temperature and washed with water for three times. The resulting aqueous and organic phases were separated. The organic phase was dried over $\mathrm{MgSO}_{4}$ and the resulting product was identified by FTIR and ${ }^{1} \mathrm{H}-\mathrm{NMR}$ spectroscopy and tested by different methods. In order to obtain optimum conditions of stirring rate, reaction time, temperature, ratio of oil: acid: peroxide, and sequence of mixing, the whole process was replicated at several new setting. In each experiment, the iodine value, epoxide content and acid value of adduct was measured. FTIR spectrum of each product was also recorded. The optimum conditions were recognized by the comparison of test results of different trials. The final obtained product of optimized conditions was characterized by FTIR and ${ }^{1} \mathrm{H}$ NMR techniques.

\section{Acetylation of epoxidized oil}

The optimum conditions due to epoxidation reaction were applied as starting point of acetylation. The recent reaction was performed by further development of epoxidation conditions at longer period of time and higher temperatures in the presence of boron trioxide catalyst. The acetylation progress was monitored and optimized by measurement of hydroxyl value, epoxy content, and recording of FTIR spectrum of separated adduct at different times. The separation was performed by washing with $\mathrm{Ca}(\mathrm{OH})_{2}$, filtering and heating for two hours. The optimum time, temperature and stirring rate were recognized by the comparison of test results of different trials. The final product was characterized by FTIR and ${ }^{1} \mathrm{H}-\mathrm{NMR}$ techniques.

\section{Larger-scale synthesis}

Large scale synthesis of acetylated sunflower oil was carried out in 5 L cylindrical reaction vessel which was equipped with an overhead stirrer and, for temperature control, a heating mantle. The employed amounts of reagents and catalysts were proportional to low scale ones. The same procedure of low scale was used for large scale synthesis. However, in this case the epoxide was not separated and the reaction was followed directly by sunflower oil to acetylated product.

\section{Equalization of gear oil Conoco}

The acetylated base oils were applied for the equalization of well-known gear oil Conoco from Conoco Phillips Company. Equalization was performed by addition of appropriate amounts of 
additives to the base oils, followed by stirring for $10 \mathrm{~min}$ at $50{ }^{\circ} \mathrm{C}$. The details of formulations as well as, physical properties of formulated oil are given in the section of results and discussion.

\section{Examination of miscibility of acetylated oil}

The probability of the mixing of the acetylated oil with some of the mineral and synthetic base oils, as well as lubricant additives was examined by mixing the appropriate amounts of the components followed by stirring for two hours. The outcomes are explained in results and discussion section.

\section{RESULTS AND DISCUSSION}

Saponification value as well as acid value of the sunflower oil is given in Table 2. The ester value which is the difference between two properties is also given. The average molecular weight of the oil can be calculated from the following equation:

Molecular weight $=(3000 \times 56.1 /$ ester value $)$

As the ester value is equal to 197.7 the obtained molecular weight will be 851.3 . By having the iodine value the number of double bonds in one gram of oil will be obtained as:

No. of double bonds in one gram of oil $=($ iodine value $/ 126.9 \times 2)$

Since the iodine value is equal to $122,4.8$ mmole of double bond exist in one gram of the oil.

The average number of double bonds in one triglyceride molecule can be calculated from the following equation. Both of the values in the numerator and de-numerator of the equation are due to one gram of oil:

Double bond per molecule $=($ no. of double bonds/no. of triglyceride mmoles $)$

Thus, the average no. of double bond per molecule will be equal to 5.6. The presence of such unsaturation in the triacylglycerol molecule, due to $\mathrm{C}=\mathrm{C}$ from oleic, linoleic, and linoleic acid moieties, functions as the active sites for various oxidation reactions. This is fairly reflected the oxidation stability of the oil. As it is indicated in Table 2, the percentage of deposit upon heating of $35 \mathrm{mg}$ of the oil at $170{ }^{\circ} \mathrm{C}$, for $50 \mathrm{~min}$ and in the air flow of $20 \mathrm{~cm}^{3} \mathrm{~min}^{-1}$ is equal to 65 which shows a weak resistance to oxidation. Oxidation is the single most important reaction of oils resulting in increased acidity, corrosion, viscosity, and volatility when used as lubricant base oils for engine oils. In many applications the fluid is required to perform at elevated temperatures and in contact with air. Consequently, in its current form, the oil does have an important disadvantage which should be overcome before usage as base stock $[2,10,14]$.

Viscosity is one of the oil's most important physical properties. It is often one of the first parameters measured by most oil analysis labs because of its importance to oil condition and lubrication. The values of viscosity at $40{ }^{\circ} \mathrm{C}$ and $100{ }^{\circ} \mathrm{C}$ (Table 2) indicate that from the aspect of this property, the oil does have acceptable values and the lubrication duties which relate to this characteristic will be done as good as mineral and synthetic oils [5, 31, 32]. Viscosity index, commonly designated VI, is an arbitrary numbering scale that indicates the change of the oil viscosity with change in temperature. Viscosity index can be classified as: low VI-below 35; medium VI: 35 to 80; high VI 80-110: very high VI: above 110 [32]. As the temperature increases, oil with low VI will lose its loading effect which results in direct contact of metallic pieces and subsequent increase of wear [33]. As it is seen in Table 2, the VI of the oil is very high. So, rheological properties of the oil will retain at elevated temperatures. 
Table 2. Comparison of some of the important properties of sunflower oil and acetylated sunflower oil.

\begin{tabular}{|l|c|c|c|}
\hline Property & \multirow{2}{*}{ Test method } & \multicolumn{2}{|c|}{ Value } \\
\cline { 3 - 4 } & & Sunflower oil & Acetylated oil \\
\hline Saponification value (mg KOH/g oil) & ASTM D-5558-95 & 199.3 & 135 \\
\hline Acid value (mg KOH/g oil) & AOCS Ca 3a -63 & 1.6 & 1.3 \\
\hline Ester value (mg KOH/g oil) & - & 197.7 & 131.1 \\
\hline Average molecular weight & - & 851.3 & 1250 \\
\hline Iodine value & AOCS Cd 1-25 & 122 & 0 \\
\hline Double bond per molecule & - & 5.6 & 0 \\
\hline Oxidation stability* & Penn State & 65 & 10 \\
\hline Vis @ $40^{\circ} \mathrm{C}$ & ASTM D-445 & 33.2 & 462 \\
\hline Vis @ $100^{\circ} \mathrm{C}$ & ASTM D-445 & 7.9 & 35 \\
\hline Viscosity index & ASTM D-2270 & 222 & 122 \\
\hline Pour point & ASTM D-92 & +3 & -9 \\
\hline Flash point & ASTM D-97 & $>350$ & 295 \\
\hline
\end{tabular}

"The percentage of insoluble deposit after 50 min heating at $170{ }^{\circ} \mathrm{C}$.

The flash point is the lowest temperature at which volumes of vapor evaporate from the oil sample to be tested under defined conditions to such an extent that together with the air over the fluid level they will form an inflammable mixture. A low flash point of oil causes high evaporation losses insofar that high local oil temperatures may occur [33]. The very high flash point of the oil designate that the usage of the oil at high temperatures does not have the firing danger.

One major characteristic of the low-temperature properties of lubricating fluids in industrial scales is pour point (PP). ASTM technique D-97 is used to determine PP. The pour point is the temperature which is $3 \mathrm{~K}$ above the temperature at which the fluid is no longer flowable under the test conditions. Oil with high PP will solidify at low temperatures, which results in a lot of harms [31]. The high pour point of sunflower oil (Table 2) is another problem which should be solved before usage. Based on the data in Table 2 and the corresponding discussion in previous paragraphs, it is clearly understood that the sunflower oil does have the difficulties of low oxidation stability and high pour point. Because of esteric characters, the low hydrolytic stability [34] is also intensifies the disadvantages. The unwanted properties of the oil can be improved by genetic modification, chemical modification, using special additives and blending $[11,14,15,35]$.

Chemical modification is the selected way which was followed in this work. This was performed by epoxidation of the oil and the subsequent acetylation of the epoxidized oil. The epoxidation was performed in the presence of the mixture of hydrogen peroxide and acetic acid as oxidant and sulfuric acid as catalyst, in dichloromethane solution. The reaction was followed in the reflux conditions at high temperatures. The reaction conditions were optimized according to time, temperature, stirring rate, amount of catalyst, the ratio of the oil to peroxide to acid, the subsequent of hydrogen peroxide and acid addition and the number of addition steps. The reaction situation was monitored by measuring iodine value, absorbance at $400 \mathrm{~nm}$, epoxy content and the comparison of relative intensities of the IR bonds. By progress of the reaction, the iodine value and absorbance showed decreasing trend, and the epoxy content showed growing trend. In the case of IR spectrum a decreasing movement was observed for the ratio of the stretching bond of unsaturated hydrogen at $3006 \mathrm{~cm}^{-1}$ to its adjacent bond (Figure 1). After optimization it was found that the temperature of $50{ }^{\circ} \mathrm{C}$, the reaction time of 9 hours, the stirring rate of $700 \mathrm{rpm}$, the catalyst amount of $0.1 \mathrm{~mL}$, ratio of oil to hydrogen peroxide to acetic acid of 2:2:1.6, firstly addition of acetic acid and secondly addition of the mixture of hydrogen peroxide and catalyst are the best conditions. The acetylation of the epoxidized oil was performed by addition of boron trioxide catalyst to the epoxide containing flask at the end of 
optimized conditions, followed by further heating and stirring. The reaction conditions were optimized according to time, temperature, stirring rate, and amount of catalyst. The reaction conditions was monitored by measuring of the epoxy content and hydroxyl value, as well as the ratio of the intensity of hydroxyl peak at $3453 \mathrm{~cm}^{-1}$ to its adjacent bond [36,37] in IR spectrum (Figure 1). It was found that the optimum conditions involve the reaction time of 5 hours, stirring rate of $700 \mathrm{rpm}$, temperature of $70{ }^{\circ} \mathrm{C}$, and the amount of catalyst of $0.2 \mathrm{~g}$ are the best conditions.

IR spectra of sunflower oil, epoxidized oil and acetylated oil are given in Figure 1. As it is shown, in the IR spectrum of the sunflower oil (Figure 1, (a)), the bond due to unsaturated hydrogen has been appeared in $3008 \mathrm{~cm}^{-1}$. The bonds related to stretching vibrations of methyl and methylene hydrogen have been emerged at 2925 and $2856 \mathrm{~cm}^{-1}$. The carbonyl bond is observed at $1745 \mathrm{~cm}^{-1}$. The bending vibrations of methyl and methylene hydrogen are seen at 1372 and $1460 \mathrm{~cm}^{-1}$. The bending vibrations of C-O bands are at 1230,1163 and $1098 \mathrm{~cm}^{-1}[13$, 38]. On the other hand, the disappearance of a peak at $3008 \mathrm{~cm}^{-1}$ (which is due to stretching of double bond $\mathrm{CH}[39,40])$ in the IR spectrum of epoxidized oil fairly confirms the formation of epoxy (Figure 1, (c)). The appearance of a peak at $943 \mathrm{~cm}^{-1}$ is the further confirmation of the recent happening. On the other hand the emergence of OH peak in $3453 \mathrm{~cm}^{-1}$ in the IR spectrum of acetylated oil fairly confirms the hydroxyl acetyl formation. The broadening of carbonyl peak at $1746 \mathrm{~cm}^{-1}$ is an additional verification of acetyl formation [37, 38].

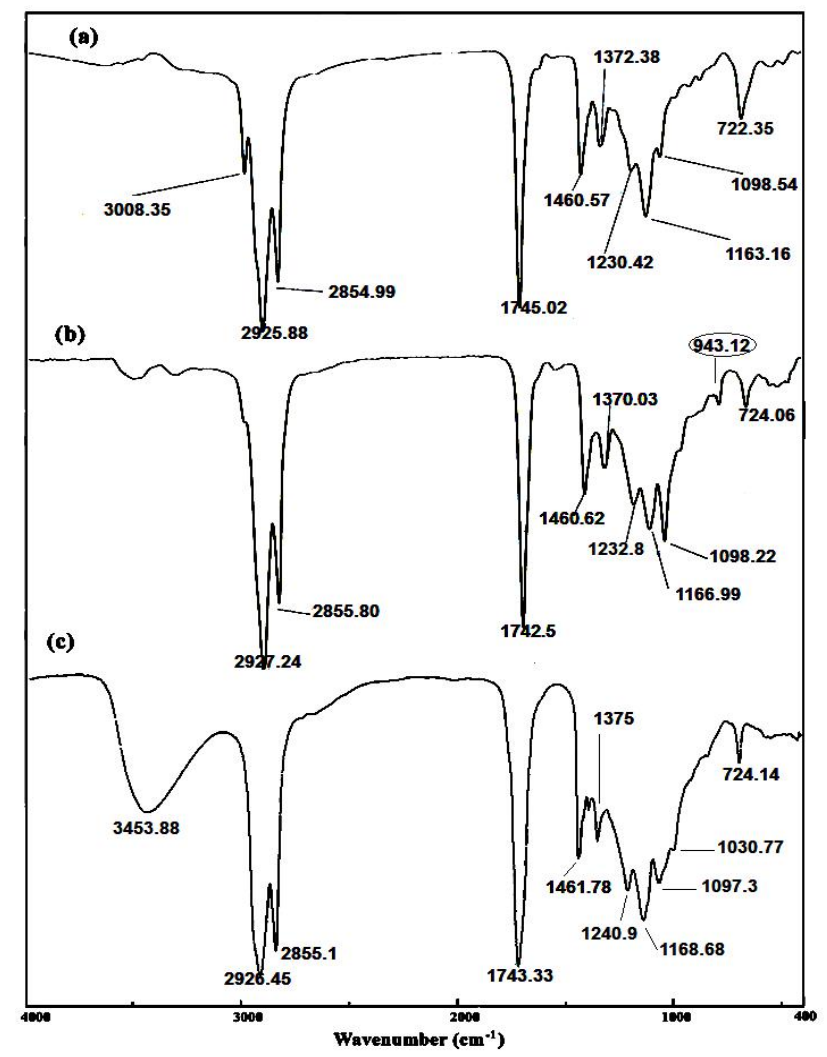

Figure 1. FTIR spectra of sunflower oil (a), epoxidized oil (b), and acetylated oil (c).

Bull. Chem. Soc. Ethiop. 2017, 31(1) 
${ }^{1} \mathrm{H}$ NMR spectra of sunflower oil, deoxidized oil, and acetylated oil are shown in Figure 2. In the sunflower spectrum (Figure 2 (a)), the observed chemical shift at 5.2-5.4 ppm can be assigned to unsaturated hydrogen of ester chains as well as $\mathrm{CH}$ hydrogen of glycerol. The chemical shift at 4.1-4.3 ppm can be related to $\mathrm{CH}_{2}$ hydrogen which is located between the double bonds. The peaks at 2.3 can be attributed to the methylene adjacent to carbonyl groups. The peaks due to further $\mathrm{CH}_{2}$ groups are seen at 1.2-1.5 ppm. The corresponding peaks of methyl hydrogen are appeared at chemical shift of below $1 \mathrm{ppm}$. Comparison of NMR spectrum of epoxidized oil (Figure 2 (b)), with unepoxidized one (Figure 2, (a)), indicate that the intensity of the peaks due to unsaturated hydrogen at $5-5.5 \mathrm{ppm}$ has considerablybeen reduced (the remained intensity is due to $\mathrm{CH}$ hydrogen of glycerol) which indicate that the double bond has been saturated. The reduction of the intensity of the peaks at $2.7 \mathrm{ppm}$ is a further confirmation of saturation. The appearance of new peaks at $5.7 \mathrm{ppm}$ in the NMR spectrum of acetylated compound (Figure 2, (c)) can be assigned to the $\mathrm{CH}$ hydrogen which is adjacent to acetyl group.

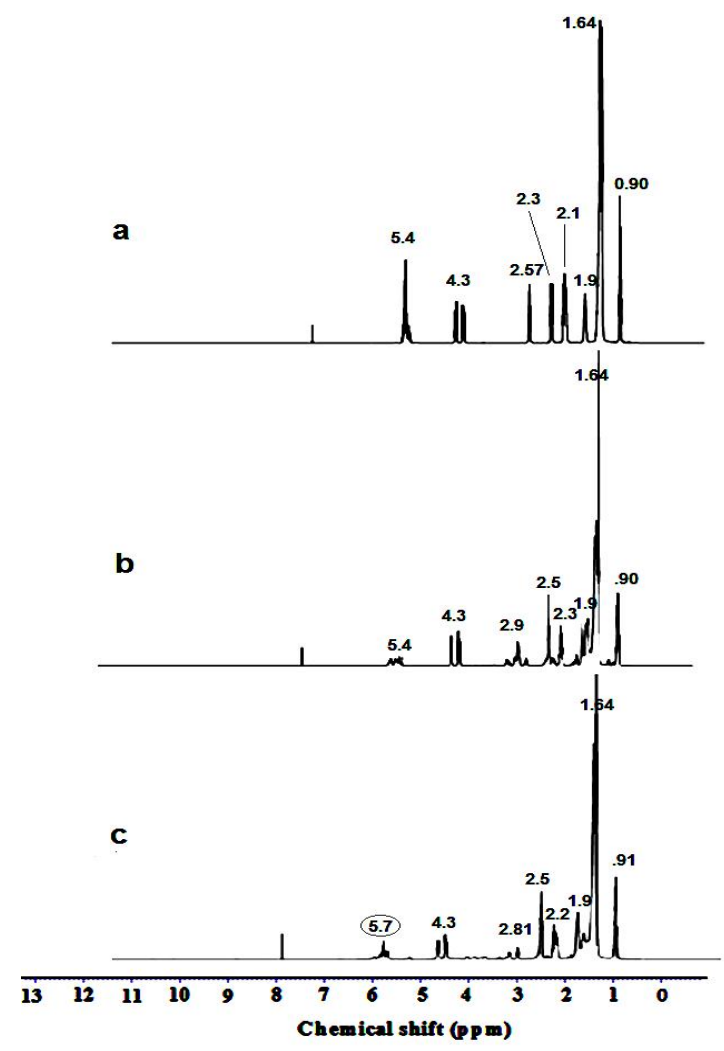

Figure 2. NMR spectra of sunflower oil (a), epoxidized oil (b), and acetylated oil (c).

Different physical properties of sunflower oil as well as chemically modified oil are compared in Table 2. The data indicate that saponification value of the oil has been decreased which indicate that the average molecular weight of the obtained adduct is more than initial sunflower oil. Interestingly, the difference between the obtained molecular weight and the corresponding value due to initial oil (i.e. 1250-850) is equal to average double bond per 
molecule (5.6) multiplied by 76. As the recent value is the sum of the hydroxyl and acetyl weights. It can be concluded that each of the double bonds has been substituted by one hydroxyl and one acetyl group. The diminishing of iodine value from 122 to 0 is further confirmation of the recent conclusion. On the other hand the considerable decrease of insoluble deposit after 50 min heating at $170{ }^{\circ} \mathrm{C}$ [38], indicate that upon acetylation the oxidation stability has been improved. This fairly can be related to the saturation of double bonds. The increase in viscosity can be assigned to the increase of steric hindrance [13] between the modified molecules relative to unmodified ones. The lowering of the pour point can also be attributed to the recent agent. The increase of complexity of the structure of the molecule upon acetylation is another reason of pour point depression [30, 39-41].

The modified sunflower oil was employed for the equalization of gear oil Conoco from Conoco Phillips Company. The details of formulation as well as the comparison of physical properties of the formulated oil with those of the reference oil are given in Table 3 . As it is seen the rheological properties of the formulated oil are close to the reference oil. In addition, the new oil does have the advantages of higher flash point, higher viscosity index and lower pour point. The FZG test evaluates fluid lubricating and wears protection properties at the interface of a loaded set of gears. Timken load is a quantitative measure that indicates the possible performance of EP additive in lubricating oil [42]. The results of both of these important tests on the gear oil formulation based on acetylated sunflower oil are acceptable (Table 3). Moreover, the weight ratio of 30:0.5:0.6 was employed for base oil (acetylated sunflower oil) and additives (HiTECH 9310 and pour point depressant Viscoplex 4-677).

Table 3. Equalization of gear oil coconut from coconut Phillips Company by the acetylated sunflower oil.

\begin{tabular}{|l|c|c|c|c|c|}
\hline Formulation & Property & Test method & \multicolumn{2}{|c|}{ Value } \\
\hline Component & $\begin{array}{c}\text { Wt. } \\
(\mathrm{kg})\end{array}$ & & & $\begin{array}{c}\text { New and used } \\
\text { formulated oil }\end{array}$ & Conoco \\
\hline Acetylated oil & 30 & Vis@40 ${ }^{\circ} \mathrm{C}$ & ASTM D-445 & 489 & 460 \\
\cline { 3 - 6 } & & Vis@100 ${ }^{\circ} \mathrm{C}$ & ASTM D-445 & 38 & 30.4 \\
\cline { 3 - 6 } & & Viscosity index & ASTM D-2270 & 122 & 96 \\
\cline { 3 - 6 } & & Flash point & ASTM D-92 & 295 & 254 \\
\cline { 3 - 6 } & Pour point & ASTM D-97 & -21 & -15 \\
\cline { 3 - 6 } & & Specific gravity & ASTM D-1298 & $0.9855(8500)$ & 0.8940 \\
\hline HiTEC 9310 & 0.5 & FZG load & ASTM D-5182 & $>12$ & $>12$ \\
\hline $\begin{array}{l}\text { Pour point depressant } \\
\text { Viscoplex 4-677 }\end{array}$ & 0.6 & Timken load & ASTM D-2782 & 62 & 60 \\
\cline { 3 - 6 } & & Color & & 4.0 & 5.5 \\
\hline
\end{tabular}

Table 4. Comparison of the lubrication properties of the chemically modified oil, SN-150 and the $95 \%$ blend of modified oil: $\mathrm{SN}-150$.

\begin{tabular}{|c|c|c|c|c|}
\hline Property & Test method & \multicolumn{2}{|c|}{ Value } & Blend \\
\cline { 3 - 5 } & & Modified oil & SN-150 & 36 \\
\hline Vis at $40^{\circ} \mathrm{C}$ & ASTM D-445 & 462 & 33.3 & 6 \\
\hline Vis at $100^{\circ} \mathrm{C}$ & ASTM D-445 & 35 & 5.61 & 109 \\
\hline Viscosity index & ASTM D-2270 & 122 & 105.9 & -15 \\
\hline Pour point & ASTM D-92 & -9 & -15 & 210 \\
\hline Flash point & ASTM D-97 & 295 & 200 & 0.8767 \\
\hline Density & ASTM D-1298 & 0.9884 & 0.8700 & \\
\hline
\end{tabular}

SN consists of solvent refined paraffinic oils that are processed to meet specific targets for saturates and sulfur content. This ensures good solubility characteristics for additive in product formulations and good antioxidant properties for high temperature applications [42]. These base oils have high VI and low pour points for good low temperature performance. The number 
beside SN represents the SUS viscosity measured in Sybolt Universal Seconds at $40{ }^{\circ} \mathrm{C}$. The results of blending of acetylated sunflower oil with solvent neutral 150 (SN 150) which is a mineral oil are given in Table 4. Although after combination, minor changes in physical properties have been occurred, the miscibility indicates that useful advantages of chemically modified oil can be added to mineral oil. The attempt for making the blends with higher percentages of acetylated oil was unproductive. Moreover, the effort for the blending of the acetylated oil with synthetic base oils such as polyalphaolephin-100 (PAI-100), polyalphaolephin-6 (PAO-6), polyisobutene (PIB) was unsuccessful and in all cases upon mixing a turbid mixture was obtained. Also, the combination of the oil with VI improving additives such as Viscoplex 4-677 and Lubrizol 7075 was failed and in both cases two phase immiscible mixtures were obtained.

\section{CONCLUSION}

In this study according to the obtained results, boron trioxide $\left(\mathrm{B}_{2} \mathrm{O}_{3}\right)$ is found to be an effective catalyst for direct acetylation of sunflower oil. Acetylation of sunflower oil causes considerable increase in viscosity. However, after following the recent reaction viscosity index is diminished. Upon chemical modification, the disadvantage of high pour point and low oxidation stability of sunflower oil will considerably be improved. Acetylated sunflower oil can successfully be employed for the formulation of gear oil. The resulting oil not only does have the specification of equalized reference oil, but also possesses the advantages such as better viscosity index, higher flash point and lower pour point. The modified sunflower oil does have a good potential for blending with mineral oil such as SN-150. This provides the conditions for development of lubrication properties of mineral oils. Blending of modified oil with synthetic base stocks such as PAO and PIB is impossible. However, mixing with other ones may be practical. In order the aim of easy and economic chemical modification, to be reached, investigation on the effect of different catalysts should be followed.

\section{ACKNOWLEDGEMENTS}

The financial support of this work by research council of Shahrekord University is highly appreciated. The authors were also partially supported by the Center of Excellence for Mathematics, University of Shahrekord.

\section{REFERENCES}

1. Shahidi, F. Bailey's Industrial Oil and Fat Products: Industrial and Nonedible Products from Oils and Fats, Vol. 6, John Wiley and Sons: Estados Unidos; 2005.

2. Randles, S.J.; Wright, M. Environmentally considerate ester lubricants for the automotive and engineering industries. J. Syn. Lub. 1992, 9, 145-161.

3. Battersby, N.S.; Pack, S.E.; Watkinson, R.J. A correlation between the biodegradability of oil products in the CEC L-33-T-82 and modified Sturm tests. Chemosphere 1992, 24, 19982000.

4. Lamboni, C.; Kétévi, A.; Awaga, K.; Doh, A. A study of heated vegetable oils used by street vendors in frying foods in Lome, Togo. Bull. Chem. Soc. Ethiop. 1999, 13, 173-178.

5. Sharma, B.K.; Adhvaryu, A.; Erhan, S.Z. Synthesis of hydroxyl thio-ether derivatives of vegetable oil. J. Agric. Food Chem. 2006, 54, 9866-9872.

6. Erhan, S.Z.; Perez, J.M. (Eds.) Biobased Industrial Fluids and Lubricants, AOCS Press: Champaign, II; 2002.

7. Campanella, A.; Rustory, E.; Baldessari, A.; Baltanas, M.A. Lubricants from chemically modified vegetable oils. Biores. Technol. 2010, 101, 245-254. 
8. Erhan, S.Z.; Sharma, B.K.; Liu, Z.; Adhvaryu, A. Lubricant base stock potential of chemically modified vegetable oils. J. Agric. Food Chem. 2008, 56, 8919-8925.

9. Lee, K.W.; Hailan, C.; Yinhua, J.; Kim, Y.; Chung, K. Modification of soybean oil intermediates by epoxidation, alcoholysis and amidation, Korean J. Chem. Eng. 2008, 25, 474-482.

10. Rudnick, L.R. Synthetic, Mineral Oils and Bio-Based Lubricants, CRC Press: Boca Raton; 2006.

11. Salih, N.; Salimon, J.; Yousif, E. Synthetic biolubricant basestocks based on environmentally friendly raw materials. J. King Saud Uni - Sci. 2012, 24, 221-226.

12. Bart, J.; Gucciardi, E.; Cavalloro, S. Biolubricants: Science and Technology, Woodhead Publishing: Cambridge, UK; 2012.

13. Brown, W.; Foote, C.; Iverson, B.; Anslyn, E. Organic Chemistry, Brooks-Cole Publishing; USA; 2009.

14. Tang, Y.; Chen, G.; Zhang, J.; Lu, Y. Highly active $\mathrm{CaO}$ for the transesterification to biodiesel production from rapeseed oil. Bull. Chem. Soc. Ethiop. 2011, 25, 37-42.

15. Akintayo, E.T. Synthesis and characterization of acrylated Parkia biglobosa medium oil alkyds. Bull. Chem. Soc. Ethiop. 2004, 18, 167-174.

16. Quinchia, L.A.; Delgado, M.A.; Valencia, C.; Franco, J.M.; Gallegos, C. Viscosity modification of high-oleic sunflower oil with polymeric additives for the design of new biolubricant formulations. Environ. Sci. Technol. 2009, 43, 2060-2065.

17. Quinchia, L.A.; Delgado, M.A.; Valencia, C.; Franco, J.M.; Gallegos, C. Viscosity modification of different vegetable oils with EVA copolymer for lubricant applications. Ind. Crops Prod. 2010, 32, 607-612.

18. Asadauskas, S.; Erhan, S.Z. Depression of pour points of vegetable oils by blending with diluents used for biodegradable lubricants. JAOCS 1999, 76, 313-316.

19. Erhan, S.Z.; Sharma, B.K.; Perez, J.M. Oxidation and low temperature stability of vegetable oil-based lubricants. Ind. Crops Prod. 2006, 24, 292-299.

20. Choi, U.S.; Ahn, B.G.; Kwon, O.K.; Chun, Y.J. Tribological behavior of some antiwear additives in vegetable oils. Tribol. Int. 1997, 30, 677-683.

21. Langeroodi, H.S.; Semnani, A. Behavior of esters in blend and its possible application. Afr. J. Pure Appl. Chem. 2009, 3, 241-246.

22. Mbugua, M.N.; Yusuf, A.O.; Gitu, P.M.; Bhatt, B.M. Conversion of Vernonia galamensis oil to pyridinyl-vernolamides and their antimicrobial activities. Bull. Chem. Soc. Ethiop. 2007, 21, 103-110.

23. Wamalwa, B.M.; Njuguna, E.N.; Shiundu, P.M.; Kamau, G.N. Thermal stability of Vernonia galamensis seed oil. Bull. Chem. Soc. Ethiop. 2000, 14, 161-167.

24. Semnani, A.; Langeroodi, H.S. An investigation on the behavior of solvent neutral 500 : Polyisobutene blends. Pet. Sci Technol. 2013, 31, 2089-2097.

25. Shakoori Langeroodi, H.; Semnani, A. The production of a group(III) base oil and the investigation of its polyisobutene blends. Pet. Sci Technol. 2013, 31, 2219-27.

26. Ashraafi, J.; Semnani, A.; Shakoori Langeroodi, H. The lubrication properties of binary blends of S-4: Polyisobutene and S-4: Polyalpholephine-100. Pet. Sci Technol. 2013, 31, 2056-64.

27. Shirani, M.; Semnani, A.; Langeroodi, H.S.; Haddadi, H. Relationship between flash points of some binary base oils and their constituent oils. Bull. Chem. Soc. Ethiop. 2015, 29, 229 237.

28. Rahimi, B.; Semnani, A.; Nezamzadeh-Ejhieh, A.; Shakoori Langeroodi, H.; Hakim Davood, M. Monitoring of the physical and chemical properties of a gasoline oil during its usage. J. Anal. Methods Chem. 2012, 2012, 8. 
29. Rahimi, B.; Semnani, A.; Nezamzadeh-Ejhieh, A.; ShakooriLangeroodi, H.; Hakim Davood, M. Application of ICP-OES in the comparative analysis of a used and fresh gasoline motor oil. Global J. Front. Res. 2012, 12, 9-14.

30. Hwang, H.S.; Erhan, Z. Modification of epoxidized soybean oil for lubricant formulations with improved oxidation stability and low pour point. JACOS 2001, 28, 1170-1184.

31. Morrison, R.; Boyd, R. Organic Chemistry, 6th ed., Prentice Hall: Englewood Cliffs, NJ; 1992.

32. Moritier, R.M.; Fox, F.M.; Orszulic, S.T. Chemistry and Technology of Lubricants, Springer: New York; 2010.

33. Wray, H.A. Manual on Flash Point Standards and Their Use: Methods and Regulations, Vol. 9, ASTM International: West Conshohoken, Pa.; 1992.

34. Boyde, S. Hydrolytic stability of synthetic ester lubricants. J. Syn. Lub. 2000, 16, 297-312.

35. Salih, N.; Salimon, J.; Yousif, E. Improved the Properties of Biolubricant Basestock, Lambert Academic Publishing: Germany; 2011.

36. Pavia, D.L.; Lampman, G.M.; Vyvyan, J.A.; Kriz, G.S. Introduction to Spectroscopy, 4th ed., Brooks-Cole Publishing: USA; 2009.

37. Silverstein, R.M.; Bassler, G.C.; Morrill, T.C. Spectroscopic Identification of Organic Compounds, Wiley: New York; 1981; p 196.

38. Fox, N.J.; Stachowiak, G.W. Vegetable oil-based lubricants-A review of oxidation. Tribol. Int. 2007, 40, 1035-1046.

39. Perez, J.M.; Kelley, F.A.; Klaus, E.E.; Bagrodia, V. Development and use of the PSU microoxidation test for diesel engine oils. SAE International 1987, paper 872028.

40. Galindo-Rosales, F.J.; Alves, M.A.; Oliveira, M.S.N. Microdevices for extensional rheometry of low viscosity elastic liquids: A review. Microfluid Nanofluid 2013, 14, 1-19.

41. Adhvaryu, A.; Erhan, S.Z. Epoxidized soybean oil as a potential source of high-temperature lubricants. Ind. Crops Prod. 2002, 15, 247-254.

42. Wesol, A.A.; Pirro, D.M. Lubricants Fundamentals, 2nd ed., Marcel Dekker: New York; 2001. 